\title{
¿Es el paciente inmigrante un buen candidato para diálisis peritoneal?
}

\author{
Inés Torrelles Charlez, Marta Alfonso Roigé, Ana Úson Nuño, Elena Fernández Labadía, Mª Dolores Carrera \\ Nivela, Carol Sorolla Villas, Mercè Borràs Sans \\ Hospital Universitario Arnau de Vilanova. Lleida. España
}

\section{Resumen}

Introducción: El paciente inmigrante a veces presenta barreras que provocan dudas de su inclusión en diálisis peritoneal.

Objetivo: Analizar si ser inmigrante influye en los resultados de la diálisis peritoneal.

Material y Método: 143 pacientes incidentes en diálisis peritoneal (años 2010-17). Datos demográficos, clínicos y de pronóstico (definición pobre resultado en diálisis peritoneal: peritonitis superior a media de la unidad, transferencia a hemodiálisis en primeros 6 meses y mortalidad relacionada con la técnica).

Resultados: Comparando los pacientes inmigrantes y no inmigrantes se observan diferencias: edad $(42,4$ vs 62,3 años; $p=<0,001$ ), numero de sesiones de entrenamiento ( 7,5 vs 9,$27 ; p=0,037)$, sexo ( 61,1 vs $25,2 \%$ mujeres; $p=0,002)$, nivel económico $(44,4$ vs $13,7 \% ; p=0,005)$, educación ( 16,7 vs $1,6 \% ; p=0,004)$, situación laboral $(5,6$ vs $61,3 \% ; p \leq 0,001)$ y grado de autonomía $(38,9$ vs $13,7 \%$ actividad prácticamente normal; $p=0,031$ ). Sin diferencias respecto a los datos pronósticos.

Un $25,3 \%$ de los pacientes presentan un pobre resultado en diálisis peritoneal y el resto un buen resultado. La única diferencia significativa entre ambos grupos son el número de sesiones de entrenamiento (10 sesiones vs 8,7 sesiones; $p=0,048$ ).

\section{Correspondencia: \\ Inés Torrelles Charles \\ C/ del Salze $n^{0} 7.25199$ Lleida \\ E-mail: ines_kto@hotmail.com}

En el análisis multivariante no se encontró asociación entre el pobre resultado de la técnica con ninguna de las variables introducidas en el modelo inicial, tan solo, se aproximó el grado de funcionalidad del paciente, aunque sin alcanzar significación estadística.

Conclusiones: Los pacientes inmigrantes, a pesar de condiciones sociodemográficas distintas, tienen un resultado similar a los no inmigrantes en diálisis peritoneal.

PALABRAS CLAVE: emigración e inmigración; pronóstico, diálisis peritoneal, aptitud física.

\section{Does the immigrant patient is a good candidate for peritoneal dialysis?}

\section{Abstract}

Introduction: The immigrant patient sometimes presents barriers that cause doubts about their inclusion in peritoneal dialysis.

Objective: To analyse if being an immigrant influences the results of peritoneal dialysis.

Material and Method: 143 incident patients in peritoneal dialysis (years 2010-17). Demographic, clinical and prognostic data (poor outcome definition in peritoneal dialysis: peritonitis greater than half of the unit, transfer to haemodialysis in the first 6 months and mortality related to the technique).

Results: Comparing immigrant and non-immigrant patients, differences were observed: age (42.4 vs 62.3 years, $p<0.001$ ), number of training sessions ( 7.5 vs $9.27, p=0.037)$, $\operatorname{sex}(61.1$ vs. $25.2 \%$ women, $p=0.002$ ), 
economic level ( 44.4 vs. $13.7 \%, p=0.005$ ), education (16.7 vs. $1.6 \%, p=0.004)$, employment situation (5.6 vs $61.3 \%, \mathrm{p}<0.001$ ) and degree of autonomy (38.9 vs $13.7 \%$ practically normal activity, $p=0.031$ ). No differences with respect to the prognostic data.

A $25.3 \%$ of patients present a poor result in peritoneal dialysis and the remaining patients a good result. Only significant differences between both groups were found in the number of training sessions (10 sessions vs 8.7 sessions, $p=0.048$ ).

The only factor that tends to be associated independently (multivariate analysis) to a poor result in peritoneal dialysis is the degree of functionality of the patient. No associations were found with being an immigrant patient or with other variables.

Conclusions: Immigrant patients, in spite of the different sociodemographic conditions, have a similar outcome to non-immigrant patients in peritoneal dialysis.

KEYWORDS: emigration and immigration; prognosis; peritoneal dialysis; physical fitness.

\section{Introducción}

El aumento de la población inmigrante resulta en un número creciente de pacientes con necesidad de tratamiento renal substitutivo. Las características propias de este colectivo -con más barreras de las habituales- provocan que a veces dudemos de la conveniencia de su inclusión en la técnica de diálisis peritoneal (DP).

La inclusión en DP depende de muchos factores ${ }^{1}$ y esto explica la gran variabilidad observada en los distintos centros y áreas geográficas ${ }^{2}$. En los estudios que han analizado el grado de elegibilidad del paciente ${ }^{3}$ destaca que el $78 \%$ de pacientes no tienen ninguna contraindicación médica ni psicosocial para elegir DP. Sin embargo, se observa una alta variabilidad de este porcentaje dependiendo del centro. Esto puede ser debido a diferencias de criterio en el equipo asistencial y/o diferencias en las características de los pacientes. No se ha analizado si el lugar de nacimiento de los pacientes influye en la elegibilidad de la técnica. Y, aún más importante, si influye en los resultados de la misma.

Por ello nuestro objetivo es analizar si el hecho de ser inmigrante influye en los resultados de la DP.

\section{Material y Método}

\section{Diseño:}

Estudio descriptivo, longitudinal y retrospectivo.

\section{Población y muestra:}

Se han incluido todos los pacientes incidentes en DP en el Hospital Universitario Arnau de Vilanova de Lleida entre enero de 2010 y junio de 2017. Se excluyeron los pacientes que han elegido la técnica de DP pero por diversos motivos no acaban siendo incluidos en programa domiciliario. Se define el paciente inmigrante como aquel no nacido en España y con un tiempo de residencia menor a 10 años.

A todos los pacientes en diálisis peritoneal se les explicó el estudio y se les invitó a participar de forma voluntaria en el mismo, leyendo y firmando la Hoja de Información: Consentimiento Informado.

El estudio se llevó a cabo conforme a la legislación europea y española vigente para este tipo de estudios y conforme a los principios éticos que tienen su origen en la Declaración de Helsinki.

\section{Variables}

\section{Las variables estudiadas han sido:}

Variables demográficas: edad, sexo, país de origen, estado civil (soltero, casado, divorciado y viudo), nivel socioeconómico (bajo, medio y alto) estudios (analfabeto, primarios, secundarios y universitarios) y situación laboral (inactivo, jubilado y activo).

Variables clínicas: autonomía en la técnica, grado de funcionalidad (requiere atenciones, actividad física limitada, actividad física mayor parte del tiempo y actividad física prácticamente normal), procedencia (consulta de Enfermedad Renal Crónica Avanzada [ERCA]), unidad de agudos, hemodiálisis [HD] y trasplante), fecha de inicio DP, modalidad DP, número sesiones de entrenamiento, función renal residual $(\mathrm{ml} / \mathrm{min}), \mathrm{d} / \mathrm{p}$ creatinina y ultrafiltración (UF).

Variables de pronóstico: tasa de peritonitis, tiempo transcurrido hasta la primera peritonitis, transferencia (HD, trasplante renal y exitus) y tiempo en DP.

El nivel socioeconómico de cada paciente se estableció según la media de ingresos anuales en España ${ }^{4}$. Se consideró bajo nivel socioeconómico cuando la renta era inferior al $50 \%$ de la media de ingresos anuales, medio si 
estaba entre el $50-150 \%$ y alto cuando superaba el $150 \%$.

Por ejemplo, la media de ingreso anual de 2017 fue de 26.535 euros, el paciente se clasificó como nivel bajo si ingresó menos de 13.267 euros, nivel medio entre 13.268 y 39.801 euros y alto si era más de 39.801 euros.

Definición resultado de técnica de DP: Se consideró que un paciente había tenido un resultado pobre en DP cuando presentaba uno o varios de los siguientes criterios: a) transferencia a $\mathrm{HD}$ en los primeros 6 meses $\mathrm{b}$ ) tasa de peritonitis y/o tiempo a la primera peritonitis superior a la media de nuestra unidad $y / 0$ c) fallecidos de complicación relacionada con la técnica.

\section{Análisis estadístico}

Se comprobó la normalidad en la distribución de las variables continuas mediante el test de Kolmogorov-Smirnov. Expresamos las variables cuantitativas como medias y desviaciones estándar. Las comparaciones bivariadas se realizaron mediante la prueba $t$ de Student para muestras independientes. Las comparaciones entre variables cualitativas, expresadas como frecuencia o porcentaje, se realizaron con la prueba chi-cuadrado.

Se consideró un resultado significativo cuando el valor de $p$ fue $<0,05$ (95\% de confianza).

Para la identificación de los factores que se asocian a un buen pronóstico en DP se realizó un análisis de regresión logística múltiple. EI análisis estadístico se realizó con SPSS versión ${ }^{13}$.

\section{Resultados}

La muestra total de pacientes fue de 143 , de los cuales un $12,6 \%$ eran inmigrantes. En la Tabla 1 se muestra la comparativa de las variables sociodemográficas, variables relacionadas con la técnica y resultados derivados de la misma, entre pacientes inmigrantes y no inmigrantes Destacar que no hay diferencias respecto a los datos pronósticos.
Tabla 1. Comparativa entre pacientes no inmigrantes e inmigrantes.

\begin{tabular}{|c|c|c|c|}
\hline & $\begin{array}{l}\text { No inmigrante } \\
n=125\end{array}$ & $\begin{array}{l}\text { Inmigrante } \\
n=18\end{array}$ & $\mathbf{p}$ \\
\hline Edad (años) ${ }^{a}$ & $62,3+14,2$ & $42,4+12,6$ & $0,000(*)$ \\
\hline $\begin{array}{c}\text { Sexo }(\%) \\
\text { Hombre } \\
\text { Mujer }\end{array}$ & $\begin{array}{l}74,8 \\
25,2\end{array}$ & $\begin{array}{l}38,9 \\
61,1\end{array}$ & $0,002\left(^{*}\right)$ \\
\hline $\begin{array}{l}\text { Estado civil (\%) } \\
\text { Soltero } \\
\text { Casado } \\
\text { Divorciado } \\
\text { Viudo }\end{array}$ & $\begin{array}{l}15,3 \\
78,2 \\
0,8 \\
5,6\end{array}$ & $\begin{array}{l}11,8 \\
76,5 \\
5,9 \\
5,9\end{array}$ & $0,415\left(^{*}\right)$ \\
\hline $\begin{array}{l}\text { Nivel socio-económico (\%) } \\
\text { Bajo } \\
\text { Medio } \\
\text { Alto }\end{array}$ & $\begin{array}{l}13,7 \\
57,3 \\
29\end{array}$ & $\begin{array}{l}44,4 \\
44,4 \\
11,1\end{array}$ & $0,005\left(^{*}\right)$ \\
\hline $\begin{array}{l}\text { Estudios (\%) } \\
\text { Analfabeto } \\
\text { Primarios } \\
\text { Secundarios } \\
\text { Universitarios }\end{array}$ & $\begin{array}{l}1,6 \\
55,6 \\
35,5 \\
7,3\end{array}$ & $\begin{array}{l}16,7 \\
50 \\
16,7 \\
16,7\end{array}$ & $0,004\left(^{*}\right)$ \\
\hline $\begin{array}{l}\text { Situación laboral (\%) } \\
\text { Inactivo } \\
\text { Jubilado } \\
\text { Activo }\end{array}$ & $\begin{array}{l}14,5 \\
61,3 \\
24,2\end{array}$ & $\begin{array}{l}77,8 \\
5,6 \\
16,7\end{array}$ & $0,000\left(^{*}\right)$ \\
\hline $\begin{array}{l}\text { Autónomo en técnica (\%) } \\
\text { No } \\
\text { Si }\end{array}$ & $\begin{array}{l}17,7 \\
81,5\end{array}$ & $\begin{array}{l}5,6 \\
94,4\end{array}$ & $0,386(*)$ \\
\hline $\begin{array}{l}\text { Grado de autonomía (\%) } \\
\text { Requiere atenciones } \\
\text { AF limitada } \\
\text { AF mayor parte del tiempo } \\
\text { AF prácticamente normal }\end{array}$ & $\begin{array}{l}8,9 \\
14,5 \\
62,9 \\
13,7\end{array}$ & $\begin{array}{l}5,6 \\
0,0 \\
55,6 \\
38,9\end{array}$ & $0,031\left(^{*}\right)$ \\
\hline $\begin{array}{l}\text { Procedencia (\%) } \\
\text { ERCA } \\
\text { Agudo } \\
\text { HD } \\
\text { Trasplante }\end{array}$ & $\begin{array}{l}80,5 \\
7,3 \\
8,1 \\
4,1\end{array}$ & $\begin{array}{l}77,8 \\
11,1 \\
11,1 \\
0,0\end{array}$ & 0,753 \\
\hline $\begin{array}{l}\text { Modalidad (\%) } \\
\text { CAPD } \\
\text { APD }\end{array}$ & $\begin{array}{l}58,1 \\
41,9\end{array}$ & $\begin{array}{l}66,7 \\
33,3\end{array}$ & 0,488 \\
\hline Sesiones de entrenamiento (n) ${ }^{a}$ & $9,2+3,5$ & $7,5+2$ & 0,037 \\
\hline Función renal residual $(\mathrm{ml} / \mathrm{min})^{\mathrm{a}}$ & $11,5+3,6$ & $10,5+3,5$ & 0,307 \\
\hline Tiempo en diálisis peritoneal (meses) & $27,5+25,6$ & $23,8+21,2$ & 0,554 \\
\hline D/P creatinina ${ }^{a}$ & $0,72+0,08$ & $0,74+0,1$ & 0,421 \\
\hline UF $(\mathrm{ml})^{\mathrm{a}}$ & $524,6+243,2$ & $534,8+234,2$ & 0,874 \\
\hline Sin peritonitis $(\%)$ & 74 & 89 & 0,73 \\
\hline Tiempo a $1^{\mathrm{a}}$ peritonitis (meses) ${ }^{\mathrm{a}}$ & $18,8+17,9$ & $14,5+19,1$ & 0,74 \\
\hline Tasa peritonitis (epis/pac/año) ${ }^{a}$ & $0,23+0,19$ & $0,11+0,8$ & 0,09 \\
\hline $\begin{array}{l}\text { Fin DP (\%) } \\
\text { Paso a HD } \\
\text { Trasplante renal } \\
\text { Exitus }\end{array}$ & $\begin{array}{l}23,2 \\
34,7 \\
15,3\end{array}$ & $\begin{array}{l}11,1 \\
38,9 \\
5,6\end{array}$ & 0,22 \\
\hline
\end{tabular}

AF: Actividad física, ERCA: consulta de Enfermedad Renal Crónica Avanzada, HD: Hemodiálisis, CAPD Diálisis Peritoneal Continua Ambulatoria, APD: Diálisis Peritoneal Automatizada, D/P creatinina: Dializado plasma de creatinina, UF: Ultrafiltración.

a: Media \pm DE.

${ }^{(*)} p<0.05$. 
Tabla 2. Comparativa población con pobre resultado en DP y buen resultado en DP.

\begin{tabular}{|c|c|c|c|}
\hline & $\begin{array}{l}\text { Pobre resultado } \\
n=37\end{array}$ & $\begin{array}{l}\text { Buen resultado } \\
\mathrm{n}=106\end{array}$ & $\mathbf{p}$ \\
\hline Edad (años) ${ }^{a}$ & $63,5+13,4$ & $58,53+15,9$ & 0,096 \\
\hline $\begin{array}{l}\text { Sexo }(\%) \\
\text { Hombre } \\
\text { Mujer }\end{array}$ & $\begin{array}{l}69,4 \\
30,6\end{array}$ & $\begin{array}{l}70,5 \\
29,5\end{array}$ & 0,907 \\
\hline $\begin{array}{l}\text { Inmigrante (\%) } \\
\text { No inmigrante } \\
\text { Inmigrante }\end{array}$ & $\begin{array}{l}91,7 \\
8,3\end{array}$ & $\begin{array}{l}85,8 \\
14,2\end{array}$ & 0,365 \\
\hline $\begin{array}{l}\text { Estado civil (\%) } \\
\text { Soltero } \\
\text { Casado } \\
\text { Divorciado } \\
\text { Viudo }\end{array}$ & $\begin{array}{l}8,3 \\
83,3 \\
2,8 \\
5,6\end{array}$ & $\begin{array}{l}17,1 \\
76,2 \\
1 \\
5,7\end{array}$ & 0,531 \\
\hline $\begin{array}{l}\text { Nivel socio económico (\%) } \\
\text { Bajo } \\
\text { Medio } \\
\text { Alto }\end{array}$ & $\begin{array}{l}16,7 \\
44,4 \\
38,9\end{array}$ & $\begin{array}{l}17,9 \\
59,4 \\
22,6\end{array}$ & 0,965 \\
\hline $\begin{array}{l}\text { Estudios (\%) } \\
\text { Analfabeto } \\
\text { Primarios } \\
\text { Secundarios } \\
\text { Universitarios }\end{array}$ & $\begin{array}{l}2,8 \\
58,3 \\
30,6 \\
8,3\end{array}$ & $\begin{array}{l}3,8 \\
53,8 \\
34 \\
8,5\end{array}$ & 0,965 \\
\hline $\begin{array}{l}\text { Autónomo (\%) } \\
\text { No } \\
\text { Si }\end{array}$ & $\begin{array}{l}16,7 \\
83,3\end{array}$ & $\begin{array}{l}16 \\
83\end{array}$ & 0,841 \\
\hline $\begin{array}{l}\text { Grado de autonomía (\%) } \\
\text { Requiere atenciones } \\
\text { AF limitada } \\
\text { AF mayor parte del tiempo } \\
\text { AF prácticamente normal }\end{array}$ & $\begin{array}{l}8,3 \\
19,4 \\
66,7 \\
5,6\end{array}$ & $\begin{array}{l}8,5 \\
10,4 \\
60,4 \\
20,8\end{array}$ & 0,133 \\
\hline $\begin{array}{l}\text { Procedencia (\%) } \\
\text { ERCA } \\
\text { Agudo } \\
\text { HD } \\
\text { Trasplante }\end{array}$ & $\begin{array}{l}86,1 \\
5,6 \\
5,6 \\
2,8\end{array}$ & $\begin{array}{l}78,1 \\
8,6 \\
9,5 \\
3,8\end{array}$ & 0,776 \\
\hline $\begin{array}{l}\text { Modalidad (\%) } \\
\text { CAPD } \\
\text { APD }\end{array}$ & $\begin{array}{l}58,3 \\
41,7\end{array}$ & $\begin{array}{l}59,4 \\
40,6\end{array}$ & 0,908 \\
\hline Sesiones de entrenamiento $(n)^{a}$ & $10+3,8$ & $8,7+3,17$ & $0,048\left(^{*}\right)$ \\
\hline Función renal residual $(\mathrm{ml} / \mathrm{min})^{\mathrm{a}}$ & $11,7+3,9$ & $11,3+3,5$ & 0,59 \\
\hline Tiempo en diálisis peritoneal (meses) & $28,8+24,5$ & $26,5+25,2$ & 0,635 \\
\hline
\end{tabular}

AF: Actividad física, ERCA: consulta de Enfermedad Renal Crónica Avanzada, HD: Hemodiálisis, CAPD: Diálisis Peritoneal Continua Ambulatoria, APD: Diálisis Peritoneal Automatizada.

a: Media \pm DE

${ }^{(*)} \mathrm{p}<0.05$

Un $25,3 \%(n=37)$ de los pacientes presentaban un resultado pobre en DP, obteniendo buen resultado en el resto. Al comparar ambos grupos (Tabla 2) se observa que la única diferencia significativa son el número de sesiones de entrenamiento previas a la inclusión en técnica ( 10 sesiones vs 8,7 sesiones; $p=0,048$ ).
En el análisis multivariante (Tabla 3) no se encontró asociación entre el pobre resultado de la técnicade diálisis peritoneal con ninguna de las variables introducidas en el modelo inicial, tan solo, se aproximó el grado de funcionalidad del paciente, aunque sin alcanzar significación estadística.

\section{Discusión}

Este estudio muestra que los pacientes inmigrantes tienen un resultado similar a los pacientes no inmigrantes en DP. Este resultado es muy significativo porque en los últimos años se ha registrado un aumento de la población inmigrante en nuestro país. La población inmigrante en Cataluña representaba un $2,9 \%$ del total de población en el año 2000 y supone en 2018 el 14,2\% según datos del Indescat ${ }^{5}$, este incremento también se observa en toda España ( $10,1 \%$ en 2018). Por consiguiente, se ha incrementado el número pacientes inmigrantes que precisan tratamiento renal sustitutivo. A esta población se debe asegurar -al igual que a todos los pacientes- una correcta información para poder elegir la modalidad de tratamiento sustitutivo renal. Sin embargo, hay factores sociodemográficos que pueden dificultar la información y hacernos dudar de la conveniencia de inclusión en programa de DP.

Un estudio ${ }^{6}$ ha abordado parcialmente este tema y concluye que la elegibilidad y elección de DP no se asocia con el estado socioeconómico. Sin embargo, el estado socioeconómico puede influir en barreras específicas para la elección de DP, en concreto barreras ambientales o espaciales y de apoyo familiar o social.

A pesar de estas dudas, existen pocos estudios que comparen los resultados de la población inmigrante con la población nativa y no se centran en la modalidad de DP. Un estudio previo que incluye pacientes incidentes en hemodiálisis y DP observa que los pacientes inmigran- 
Tabla 3. Análisis multivariante. Factores asociados al resultado en DP.

\begin{tabular}{|l|l|l|}
\hline & $\boldsymbol{\beta}$ & $\mathbf{p}$ \\
\hline Edad & 0,995 & 0,757 \\
\hline Inmigración & 0,986 & 0,986 \\
\hline Nivel socioeconómico bajo & 1,650 & 0,438 \\
\hline Nivel socioeconómico medio & 2,023 & 0,135 \\
\hline Grado funcionalidad requiere atenciones & 0,385 & 0,371 \\
\hline Grado Funcionalidad & 0,195 & 0,082 \\
\hline Actividad limitada & & \\
\hline Grado Funcionalidad & 0,308 & 0,140 \\
\hline Prácticamente normal & 0,931 & 0,237 \\
\hline Sesiones de entrenamiento & & \\
\hline
\end{tabular}

$\boldsymbol{\beta}: \operatorname{Exp} \beta$

p: Chi cuadrado de Pearson.

tes tienen mayor supervivencia (corregido por edad) en una población urbana holandesa sin diferenciar los resultados según la modalidad de diálisis ${ }^{7}$. Otro estudio, analiza los resultados de los pacientes pediátricos teniendo en cuenta el origen de los padres, en este caso los resultados son peores en el caso de progenitores inmigrantes ${ }^{8}$.

Nuestro estudio confirma que estos pacientes tienen determinadas barreras como puede ser una mayor proporción de analfabetismo o un nivel económico bajo. Sin embargo, estas y otras barreras, tal como hemos observado, no impiden un correcto aprendizaje de la técnica y un buen resultado de la misma. En realidad, la subpoblación de pacientes inmigrantes precisa menos sesiones de entrenamiento que los pacientes nativos. Esto puede explicarse por el efecto de la edad, puesto que son pacientes más jóvenes. En un estudio previo ${ }^{9}$ ya observamos que la edad y la comorbilidad eran los únicos factores asociados a necesitar más sesiones de entrenamiento. A destacar que los pacientes que precisaban más sesiones tenían los mismos resultados en DP que aquellos que aprendieron más rápido.

En el estudio actual, al analizar la diferencia entre los pacientes con resultado bueno o pobre en $\mathrm{DP}$, el único factor que los diferencia es el número de sesiones de entrenamiento que habían requerido. Sin embargo, cuando analizamos los factores que influyen de forma independiente en el resultado en DP, el único factor con cierta tendencia a la asociación es el estado funcional del paciente. Los pacientes con actividad física limitada en el momento de inclusión en DP tienen peor resultado en la técnica.
Este hallazgo nos confirma por una parte que las dificultades en el aprendizaje no son un motivo de peor pronóstico siempre que aseguremos un buen entrenamiento final. Por otra parte, las barreras sociodemográficas o el ser un paciente inmigrante tampoco suponen un peor resultado en DP. El buen resultado de la población inmigrante no se explica por la edad puesto que el análisis multivariante corrige por este factor. Así pues, este estudio apoya los resultados de una publicación reciente ${ }^{10}$ que preconiza que el no ser restrictivos en la inclusión de pacientes en DP no supone tener un mal resultado.

El porcentaje de pacientes inmigrantes en nuestro estudio es bajo $(12,6 \%)$ respecto la población nativa, pero es proporcional a la población actual de nuestra área. Por lo tanto, parece ser una muestra representativa. Sin embargo, los buenos resultados obtenidos en DP no son aplicables a toda la población inmigrante, al igual que a toda la población nativa, ya que previamente hay un proceso de elección de modalidad.

A pesar de que ser un paciente inmigrante puede, en algunos casos, considerarse una dificultad en la adaptación en DP, no es motivo para no dedicar esfuerzos a su inclusión y mantenimiento en programa.

En vista a estos resultados podemos concluir que los pacientes inmigrantes, a pesar de condiciones sociodemográficas distintas, tienen un resultado similar a los no inmigrantes en diálisis peritoneal.

Los autores declaran no tener conflicto de interés.

Recibido: 5 abril 2018

Revisado: 20 abril 2018

Modificado: 30 mayo 2018

Aceptado: 2 junio 2018

\section{Bibliografía}

1. Moreiras MM. De dónde venimos y a dónde vamos en diálisis peritoneal: identificando barreras y estrategias de futuro. Nefrologia (Madr.) 2014;34:756-67.

2. Lameire N. Epidemiology of Peritoneal dialysis: a story of believers and nonbelievers. Nat Rev Nephrol 2010;6(2):75-82. 
3. Mendelssohn DC, Mujais SK, Soroka SD, Brouillette J, Takano T, Barre PE et al. A prospective evaluation of renal replacement therapy modality eligibility. Nephrol Dial Transplant. 2009;24(2):555-61.

4. Datosmacro.com [Internet]. Madrid: Expansión; enero 2018 [Consultado 23 febrero 2018]. Disponible en: https://www.datosmacro.com/mercado-laboral/ salario-medio/espana.

5. Idescat.cat [Internet]. Barcelona: idescat; enero 2018 [Consultado 16 febrero 2018]. Disponible en: https://www.idescat.cat/indicadors/?id=anual$s \& n=10332 \& \mathrm{col}=1$.

6. Prakash S, Perzynski AT, Austin PC, Wu CF, Lawless $M E$, Paterson JM et al. Neighborhood socioeconomic status and barriers to peritoneal dialysis: a mixed methods study. Clin J Am Soc Nephrol. 2013; 8(10):1741-9.

7. Van den Beukel TO, Dekker FW, Siegert CEH. Increased survival of immigrant compared to native dialysis patients in an urban setting in the Netherlands. Nephrol Dial Transplant. 2008;23:3571-7.

8. Schoenmaker NJ, Tromp WF, Van der Lee JH, Adams $B$, Bouts $A H$, Collard $L$ et al. Disparities in dialysis treatment and outcomes for Dutch and Belgian children with immigrantparents. Pediatr Nephrol. 2012; 27(8):1369-79.
9. Borràs M, Sorolla C, Carrera D, Martín M, Villagrassa E, Fernández E. Patients with learning difficulties: outcome on peritoneal dialysis. Adv Perit Dial. 2006; 22:116-8.

10. DeFijter CWH, Van Diepen ATN, Amiri F, Dekker FW, Krediet RT. Patient-reported outcomes (PROs) argue against the limited use of peritoneal dialysis in end-stage renal disease. Clin Nephrol. 2018; 26.

Este artículo se distribuye bajo una Licencia Creative Commons Atribución-NoComercial 4.0 Internacional. https://creativecommons.org/licenses/by-nc/4.0/ 